\title{
Importance of machine learning for enhancing ecological studies using information-rich imagery
}

\author{
Antoine M. Dujon ${ }^{1, *}$, Gail Schofield ${ }^{2, *}$ \\ ${ }^{1}$ Centre for Integrative Ecology, School of Life and Environmental Sciences, Deakin University, Geelong, Victoria 3280, \\ Australia \\ ${ }^{2}$ School of Biological and Chemical Sciences, Queen Mary University of London, London E1 4NS, UK
}

\begin{abstract}
There is increasing demand for efficient ways to process large volumes of data from visual-based remote-technology, such as unmanned aerial vehicles (UAVs) in ecology and conservation, with machine learning methods representing a promising avenue to address varying user demands. Here, we evaluated current trends in how machine learning and UAVs are used to process imagery data for detecting animals and vegetation across habitats, placing emphasis on their utility for endangered species. We reviewed 213 publications that used UAVs at 256 study sites, of which just 89 (42\%) used machine learning to assess the visual data. We evaluated geographical and temporal trends and identified how each technology is used at a global scale. We also identified the most commonly encountered machine-learning methods, including potential reasons for their limited use in ecology and possible solutions. Thirteen out of the 17 habitats defined by the International Union for Conservation of Nature (IUCN) habitat classification scheme were monitored using UAVs, while 12 habitats were monitored using both UAVs and machine learning. Our results show that, while machine learning is already being used across many habitat types, it is primarily restricted to more uniform habitats at present. Out of 173 plant and animal species monitored using UAV surveys, 30 were of conservation concern, with machine learning being used to assess UAV imagery data for 9 of these species. In conclusion, we anticipate that the joint use of UAVs and machine learning for ecological research and conservation will expand as machine learning methods become more accessible.
\end{abstract}

KEY WORDS: Meta-analysis - Computer vision - Video analysis - Big data - Classifier · Deep learning $\cdot \mathrm{BRUVs} \cdot \mathrm{ROVs}$

\section{INTRODUCTION}

Recent advances in visual technology, both underwater (e.g. baited remote underwater video stations [BRUVs] and remotely operated underwater vehicles [ROVs]) and in the air (unmanned aerial vehicles [UAVs] and unmanned aerial systems [UASs]), are providing a completely new perspective of wildlife and the ecosystems in which they live (Mallet \& Pelletier 2014, Christie et al. 2016). For example, UAVs are being used to map habitats (Kaneko et al. 2014, Szantoi et al. 2017) as well as monitor the abundance, behaviour and health of wildlife (Barasona et al.

${ }^{*}$ Corresponding authors: antoine.dujon@yahoo.fr; gail.schofield@qmul.ac.uk
2014, Chabot et al. 2015, Christiansen et al. 2016, Schofield et al. 2017, Bonnin et al. 2018). In particular, UAVs provide an opportunity to monitor elusive animals, including vulnerable and endangered species for which data remain limited, facilitating improved conservation actions, including serving as an antipoaching tool (Mulero-Pázmány et al. 2014, Olivares-Mendez et al. 2015, Christie et al. 2016).

While the utility of UAVs as monitoring tools has been widely demonstrated across marine and terrestrial systems (e.g. Parry et al. 2003, Mallet \& Pelletier 2014, Hodgson et al. 2018), large volumes of imagery (stills or video) and sensory information must be pro-

() The authors 2019. Open Access under Creative Commons by Attribution Licence. Use, distribution and reproduction are unrestricted. Authors and original publication must be credited. 
cessed to extract relevant information, which is generally termed 'regions of interest' and sometimes 'points of interest' (Dell et al. 2014, Weinstein 2018). Imagery data is primarily extracted manually (by the naked eye), which is time consuming. Consequently, if all regions of potential interest are not extracted, the data must be viewed more than once, increasing the processing time (Dell et al. 2014, Weinstein 2018). Thus, to incorporate imagery-based technology, such as UAVs, ROVs and BRUVs, into long-term monitoring programs involving repeated surveys that generate large amounts of data requiring processing, it is essential to develop automated methods that extract regions of interest. Such methods also help standardize analysis and reduce human effort (Tarca et al. 2007, Wagstaff 2012, Thessen 2016).

This need for the rapid analysis of large imagery datasets has driven recent advances in the use of computer algorithms, including machine-learning algorithms (Domingos 2012, Dell et al. 2014, L'Heureux et al. 2017, Weinstein 2018). The core component of a machine-learning algorithm is its ability to learn how to perform important tasks for a given set of data by generalizing information from a relatively large number of samples (Fig. 1). For example, a machine-learning algorithm that is trained to identify regions of interest on imagery data automatically learns a set of rules to separate the regions of interest from the background in a given sample (Domingos 2012, Weinstein 2018). In complex, real-life situations that are commonly encountered in ecology, and when large amounts of data must be processed, is it often easier to train a machine-learning algorithm by showing it examples of desired inputs-outputs, rather than manually programming a set of rules by attempting to anticipate the desired response for all possible inputs (Fig. 1; Jordan \& Mitchell 2015). Thus, machine-learning algorithms are excellent candidates for automating a range of time-consuming tasks (Domingos 2012, Weinstein 2018). However, machine learning is not yet systematically used to process imagery data in the field of ecology (Thessen 2016), with some pioneering studies existing in behavioural biology (e.g. Guilford et al. 2009, Egnor \& Branson 2016) and conservation biology (e.g. Kampichler et al. 2010, Tabak et al. 2018). Therefore, it is important to identify why gaps exist in the use of machine learning to support the development on the use of imagery-based monitoring in ecological studies.

Here, we conducted a literature review to establish how UAVs and machine learning are currently being used in the field of ecology and what issues are currently limiting their widespread integration in re-

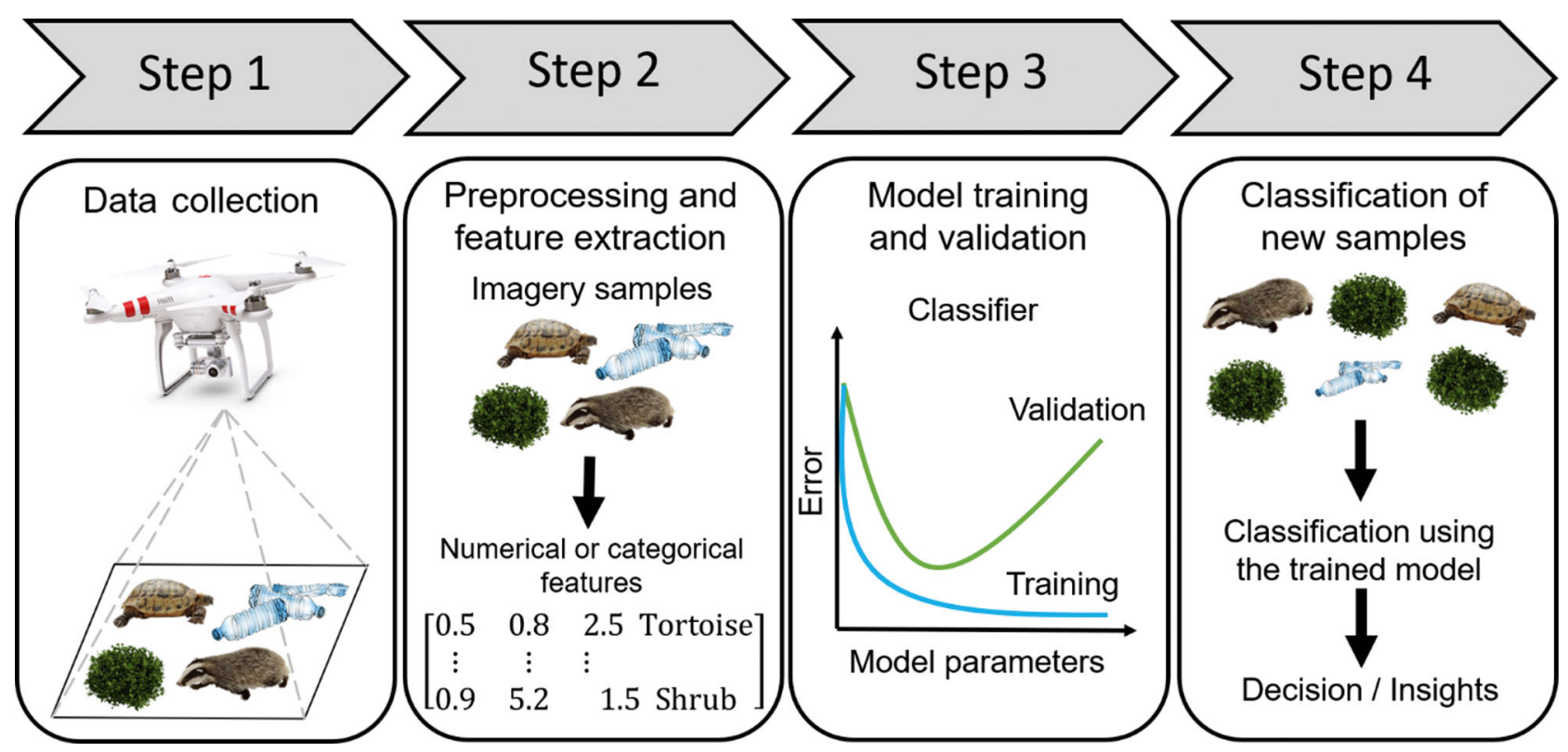

Fig. 1. To generate a machine learning algorithm, 4 main steps are usually followed. When using UAVs, these 4 steps are: Step 1: Data collection, during which an operator collects a large amount of imagery data. Step 2: Data pre-processing, during which selected features are extracted from the imagery data for use in training a model. Step 3: The training and validation of a machine learning algorithm, during which the model is selected following a series of iterations where it is trained and tested multiple times to optimize its performance. Step 4: Once the model performance is satisfactory, the model is routinely applied on newly collected data, and classification results are used to generate new insights on the system or species of interest 
search and conservation. Specifically, we (1) documented the landscape types (habitat and ecosystem) in which UAV surveys are used to monitor both vegetation and animals, including endangered species; (2) identified potential gaps in habitat/ecosystem coverage and/or associated with wildlife being monitored with UAVs; (3) identified potential biases in global regions where UAV surveys are currently being conducted; $(4)$ identified in which cases machinelearning algorithms are being used; and (5) identified the underlying reasons for this use. The use of UAVs in ecology is relatively new (Koh \& Wich 2012, Christie et al. 2016), with machine learning currently increasing in popularity across many scientific fields (Jordan \& Mitchell 2015, L'Heureux et al. 2017). Thus, we hypothesized that a bias would exist towards using UAVs to detect larger animals (more easily detectable) and more uniform (homogeneous) aquatic and landscape types, with a similar pattern being observed when UAVs are used in conjunction with machine learning (Dell et al. 2014, Weinstein 2018). We also hypothesized that there would be publication bias towards organisations in countries with an advanced economy for both UAVs and the implementation of machine-learning tools (United Nations Educational Scientific and Cultural Organization 2016). This review is expected to demonstrate the accessibility and potential of machine learning linked with UAV technology to the field of ecology, highlighting potential obstacles limiting its more widespread use, as well as its potential for use in long-term and routine monitoring practices and conservation management efforts of vulnerable and endangered species.

\section{MATERIALS AND METHODS}

\subsection{Literature review}

We assembled data from published sources on ecological studies using UAVs with and without machine learning. We searched the Thomson Reuters ISI Web of Science ${ }^{\mathrm{TM}}$ database and Google Scholar for papers that included a combination of one of the following terms: 'drone' or 'unmanned aerial vehicle' or 'unmanned aerial system' or 'unoccupied aircraft system' or 'remotely piloted aerial system' or their abbreviations with any of the search terms 'wildlife' or 'ecology' or 'conservation' or 'behaviour' or 'behavior' and 'machine learning', in the topic field, which includes the title, abstract, keywords and Keywords Plus (i.e. words that frequently appear in the titles of the articles cited within a publication). To locate additional articles that might not have been identified by the initial search, we also checked the reference lists of relevant papers based on the pre-defined keywords. In total, we identified 213 publications since 2004 up to the end of 2018 (i.e. 31 December) that met the keywords-based criteria (see Supplement 1 at www.int-res.com/articles/suppl/n039p091_supp1. xlsx). The year 2004 corresponded to the year of publication of the oldest study that was found and that met the criteria.

To identify temporal trends, for each article, we documented the year and journal of publication and determined the annual number of publications over the 2004-2018 period. When available, we determined the impact factor of the journal of publication using InCites Citation Reports website (https:// clarivate.com/products/incites/). In ecology, the first author is often viewed as the scientist taking full responsibility for the publication (Duffy 2017). Therefore, for each publication, we determined the university with which the first author was affiliated and the country in which that university was based. We then determined the number of publications associated with each country using the university of affiliation of the first authors. Furthermore, the country of affiliation was also classified based on its current level of economic development, as either having an 'advanced economy' or 'developing economy' following the 2015 World Economic Outlook assessment appellation and classification of country economies (International Monetary Fund 2016). Economic country classification by the International Monetary Fund is based on a range of indicators, such as the per capita income level, the export diversification and the degree of integration into the global financial market (see International Monetary Fund 2016 for full details on the classification of economies). Overall, the economic investment in scientific research tends to be greater in countries with an advanced economy compared to countries with a developing economy (Mazloumian et al. 2013, International Monetary Fund 2016), and this difference might impact how UAVs and machine learning technologies are used. For instance, countries with an advanced economy might be more likely to use UAVs and machine learning to study species of conservation concern compared to countries with a developing economy.

For each publication, we determined (1) the ecosystem type (terrestrial, marine or freshwater) and (2) the habitat type(s) following the highest classification level of the International Union for Conservation of Nature (IUCN) Classification Scheme, which defines 
17 main habitats (International Union for Conservation of Nature 2012). We also determined (3) the number and the geographical coordinates of all study sites (i.e. latitude and longitude). If the exact coordinates were not available, we made an approximation using Google Earth for each site based on information provided in the publication. We determined (4) the study species and their IUCN Red List category at the global level when available (using the IUCN database at www.iucnredlist.org). Using this information, we determined the number of publications regarding each ecosystem, habitat type and the number of species in each of the 8 categories of the IUCN Red List.

To quantify the size of the different regions of interest covered by the articles, we first defined 4 categories: (1) 'body size', when the focus was on detecting individuals of a given species (vertebrate or invertebrate) and when the single bodies were clearly identifiable, (2) 'plant size,' when the focus was on detecting individual plants, (3) 'nest size,' when the focus was on detecting individual animal nests (of birds, reptiles, or mammals) to quantify the reproduction effort or as evidence of the presence of an animal species in a given area (e.g. for elusive species), which is important for species where documenting actual individuals is difficult, including threatened species, such as sea turtles, crocodilians and various bird species (e.g. Schofield et al. 2017, Bonnin et al. 2018), and (4) 'feature size,' when the focus was on detecting clusters of dense vegetation or aggregated invertebrates in which individuals are difficult to distinguish from each other (e.g. seagrass, moss and aquatic vegetation beds, coral reefs, stromatolites, mussel beds or homogeneous sections of habitat). For the 'body size' grouping, we determined the body size of the detected vertebrates, either from the article or from other peer-reviewed studies on the same species (assuming average adult body size, unless otherwise stated in the original article, see 'Data' in Supplement 1 for all database references). For the 'plant size' grouping, we determined the diameter of the individual plant canopy, using measurements provided in the articles. For the 'feature size' grouping, we determined the typical width of a feature from the article. Finally, for the 'nest size' grouping, we determined the diameter of the nests from the article or from other publications describing the nests of the same species (see 'Data' in Supplement 1). We relied on nest or body size estimates obtained from other peer-reviewed studies in a total of 87 publications. This approach of using size estimates when exact measurements were not available allowed us to compare the relative magnitude of size ranges for the different regions of interest in each of the 4 categories.

Finally, for each study site, we determined whether the authors used machine learning to detect regions of interest, and we established a list of the most commonly used machine-learning methods in drone ecology. In parallel, we generated an estimate of the popularity and usefulness of the machine-learning methods by entering the name of each method in the topic field of the Thomson Reuters ISI Web of Science $^{\mathrm{TM}}$ database to obtain the annual number of publications in which it was used for 2004-2018 across all scientific fields.

\subsection{Data consolidation and statistical analyses}

To investigate whether the size of the regions of interest influenced the probability that machine learning was used in a publication, we fitted a series of Bayesian logistic regression models to the data (see Supplementary Methods in Supplement 2 at www.int-res.com/articles/suppl/n039p091_supp2.pdf for equations).

To investigate potential differences between individual body size, individual plant size, feature cluster size and nest size and the probability that machine learning was used, we started by separately fitting a model to each group. Then, we fitted an additional 3 Bayesian logistic regression models by cumulatively adding each group to the explanatory variable of the model (i.e. the first model only includes body size as the explanatory variable, the second model includes both body size and plant size, the third model includes body size, plant size and feature size, and the fourth model includes all 4 groups). Only 3 publications surveyed $>1$ ecosystem (maximum of 2 ecosystems) $(<1.5 \%$ of the total number of publications). Seven publications $(<3.5 \%$ of the total number of publications) surveyed $>1$ habitat (maximum of 2 habitats). As the number of publications with repeated measurements at the ecosystem or habitat level was relatively low, they were analysed in a similar way to the other publications when fitting the Bayesian logistic regression models.

Throughout this manuscript, 2 proportions or 2 means were considered significantly different if their $95 \%$ credible intervals (CI) did not overlap. The coefficients of the logistic regression models were considered to be statistically significant if their 95\% CI did not include 0 . We report all of the estimated parameters followed by their 95\% CI between square brackets. The details of the prior distributions 
that we used are provided in Supplementary Methods in Supplement 2.

All Bayesian statistical analyses were performed using the RStan package (Stan Development Team 2018) within the R software v.3.3.2. (R Development Core Team 2013). Geographical data were assimilated using Quantum-GIS software v.2.18 (QGIS Development Team 2015).

\section{RESULTS}

\subsection{Publication trends and geographic trends in author origin and study site location}

Of the 213 ecological studies identified using UAVs, 89 publications used machine learning. The first study using UAVs was published in 2004 by Trathan (Trathan 2004). Interestingly, this study was also the first to combine UAVs and machine learning. Of note, $197(93 \%)$ of all studies with and without machine learning were published from 2012 to 2018 (Fig. 2c). The percentage of publications using UAVs and machine learning was $\sim 40 \%$ over the past $4 \mathrm{yr}$ (2014-2018).

For the 213 ecological studies identified using UAVs, first authors were mostly affiliated with universities located in countries with an advanced economy $(92 \%, \mathrm{n}=195$ publications (Fig. 2a,b,d). A total of $89(42 \%)$ of these 213 publications used machine learning to process UAV data, of which 81 (90\%) were, again, from North America (Fig. 2d).

From the 213 publications, the coordinates of 256 study sites were delineated (Fig. 2a). Of these, $72 \%$ were in countries with an advanced economy ( $\mathrm{n}=$ 184 sites). Machine learning was used to process UAV data from 110 of 256 of the study sites, of which $82(75 \%)$ were from North America, Europe and Oceania (Fig. 2e). In addition, a total of $4 \%$ of the study sites $(n=9)$ were located in Antarctica (Fig. 2a).

There was no significant difference in the average journal impact factor of articles that presented UAV data without machine learning (average impact factor of $2.88[2.46,3.12])$ versus with machine learning (average impact factor of $2.83[2.32,3.10]$ ).

\subsection{Ecosystems surveyed}

Most UAV studies were conducted in terrestrial ecosystems ( $\mathrm{n}=97$ publications), followed by marine ecosystems ( $\mathrm{n}=74$ publications) and freshwater ecosystems ( $\mathrm{n}=45$ publications; Fig. 3a). For each sys- tem, machine learning was used to assess imagery data from just under half of the UAV studies $(45 \%$ of studies [36\%, $56 \%$ ] for terrestrial, $35 \%$ [25\%, $46 \%$ ] for marine and $42 \%$ [29\%, $57 \%$ ] for freshwater ecosystem), with no significant difference between the ecosystem types.

\subsection{Habitats surveyed}

All but 4 of the 17 habitats defined in the highest level of the IUCN habitat classification scheme were covered by at least 1 UAV study (Fig. 3b, see also Supplementary Results in Supplement 2). Of the UAV studies, $72 \%$ (154 of 213 publications) were conducted in 5 habitats: marine neritic (i.e. subtidal), wetlands, artificial terrestrial (here artificial in the sense of a heavily modified and/or degraded habitat by human activities), marine coastal and supratidal and forest (Fig. 3b). The 5 least covered habitats (desert, aquatic and artificial, marine intertidal, savanna and 'other' habitat, such as Antarctica) accounted for just $10 \%(\mathrm{n}=22)$ of publications (Fig. $3 \mathrm{~b})$. The 4 remaining habitats (introduced vegetation, shrubland and grassland) accounted for $20 \%(\mathrm{n}=43)$ of publications. We were not able to determine the habitat for 1 publication in the terrestrial ecosystem.

Machine learning was primarily used to analyse UAV data from 4 habitats: artificial terrestrial, wetlands, introduced vegetation and marine coastal and supratidal for a total of $42 \%(n=37)$ of the 89 studies (Fig. 3b, Supplementary Results 1).

\subsection{Wildlife and plant species monitored}

\subsubsection{Animals}

A total of 129 animal species were identified from 117 publications (Fig. 4a, 'Data' in Supplement 1). Machine learning was used in $15 \%(n=18)$ of these publications. Of these, $29(23 \%)$ species were categorised as vulnerable, endangered or critically endangered by the IUCN Red List (Fig. 4a). Machine learning was used to detect $8(30 \%)$ of these 29 species (Fig. 4a).

Most birds and mammals were surveyed with UAVs in terrestrial and marine ecosystems and to a lesser extent in freshwater ecosystems. Reptiles were only surveyed in marine and freshwater ecosystems. Fishes were surveyed to similar levels in both marine and freshwater ecosystems. UAVs were primarily used to detect individual animals or nests and were 

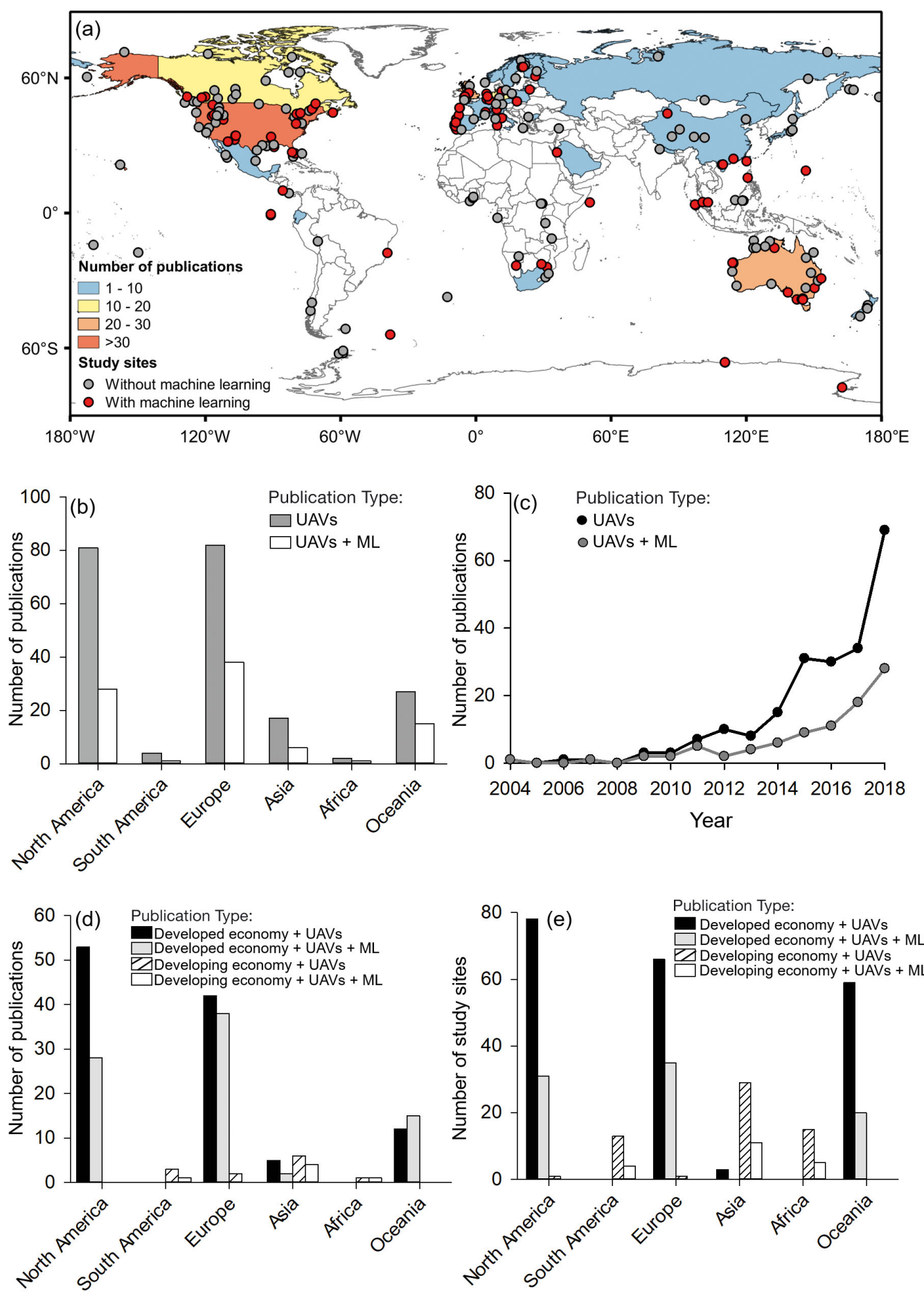

Fig. 2. (a) Geographical distribution of the university affiliation of the first author by country (shown as number of publication groupings) and distribution of the study sites $(\mathrm{n}=256)$ where machine leaning was used to support UAVs $(\mathrm{n}=110)$ and was not used ( $\mathrm{n}=146)$. (b) Number of ecology publications in which UAVs were used alone or in combination with machine learning (ML) grouped by continents based on the university of affiliation of the first author. (c) Increase in the number of ecology publications in which UAVs were used alone $(n=213)$ or in combination with machine learning $(n=89)$ from 2004 to 2018 . (d) Number of ecology publications in which the first author was affiliated to a university located in a country with an advanced or developing economy and for which UAVs were used alone or in combination with machine learning. The data are grouped by continents based on the university of affiliation of the first author. (e) Number of study sites located in a country with an advanced or developing economy and for which UAVs were used alone or in combination with machine learning. The data is grouped by continents based on location of the study sites 
Table 1. Summary of the most commonly used machine-learning methods encountered in ecology to process imagery data collected using UAVs and the main R and Python packages to implement them

\begin{tabular}{|lcccl}
\hline Machine-learning algorithm & $\begin{array}{c}\text { Number of } \\
\text { publications }\end{array}$ & $\begin{array}{c}\mathrm{R} \\
\text { packages }\end{array}$ & $\begin{array}{c}\text { Python } \\
\text { packages }\end{array}$ & Useful sources \\
\hline Object based image analysis & 22 & SegOptim & RSGISLib & Blaschke (2010) \\
Random forest & 16 & rf & scikit-learn & Cutler et al. (2007), Strobl et al. (2009) \\
Maximum likelihood classification & 13 & rasclass & mlpy & Foody et al. (1992) \\
Support vector machine & 12 & e1071 & scikit-learn & Hsu et al. (2008), Mountrakis et al. (2011) \\
K-means clustering & 9 & stats & scikit-learn & Olden et al. (2008) \\
Convolutional artificial neural network & 7 & keras & tensorflow, keras & Chollet (2015), Wu (2017) \\
Thresholding & 5 & imager & OpenCV & Zou et al. (2016) \\
\hline
\end{tabular}

rarely used to survey clusters of animals ('Data' in Supplement 1 and Supplementary Results in Supplement 2). The individual body size of animals detected using drones ranged from 0.15 to $26.00 \mathrm{~m}$, with a median body size of 1.00 m (Fig. 5a, see also Supplementary Results in Supplement 2). Animal nests were detected in all 3 ecosystems, with a diameter ranging from 0.07 to $2.50 \mathrm{~m}$ and a median nest diameter of $0.97 \mathrm{~m}$ (Fig. 5d).

Individual animals for which individual bodies were detected were surveyed in 11 of the 17 habitats (Fig. 3b, see also Supplementary Results in Supplement 2). Machine learning was used to analyse UAV data of animals surveyed in 9 habitats and has been used at similar levels in the marine neritic, marine coastal and supratidal habitats and artificial terrestrial savanna habitat (Fig. 3b). The estimated body size of individual animals detected using machine learning ranged between 0.22 and $3.98 \mathrm{~m}$, with a median body size of $0.88 \mathrm{~m}$ (Fig. 5a). Animal nests were primarily surveyed in 6 habitats, with forests being the most represented. The estimated nest size ranged from 0.07 to $0.35 \mathrm{~m}$, with a median nest size of $0.21 \mathrm{~m}$ (Fig. 5d, Supplementary Results in Supplement 2). Two of these publications used machine learning, using an estimated individual nest size of $0.07 \mathrm{~m}$, while one in the marine coastal and supratidal habitats used an estimated nest size of $0.35 \mathrm{~m}$.

\subsubsection{Plants}

The number of habitat-based surveys in which UAVs were used to detect vegetation $(\mathrm{n}=92)$ was smaller compared to surveys in which animals were detected $(\mathrm{n}=117)$. However, machine learning was used in $70 \%(n=64)$ of these 92 publications, which is significantly more compared to animals. We identified a total of 44 plant species in the 92 publications, of which only 1 plant species was classified as vulner- able by the IUCN Red List (Fig. 4b). Machine learning was used to detect that species.

Vegetation was surveyed predominantly in the terrestrial ecosystem and, to a lesser extent, in the freshwater and marine ecosystems (Fig. 3d). Vegetation was also surveyed in 12 of the 17 habitats, with the artificial terrestrial, forest, wetlands and introduced vegetation habitats being the most represented. UAVs were mostly used to detect clusters of vegetation, rather than individual plants (Fig. 3d, see also 'Data' in Supplement 1).

Machine learning was used to detect individual plants or clusters of vegetation from UAV data in 11 habitat types (Fig. 3d, see also Supplementary Results in Supplement 2). Machine learning was also used to distinguish individual plants with a canopy diameter ranging from 0.25 to $45.0 \mathrm{~m}$, with a median diameter of $4.0 \mathrm{~m}$ (Fig. 5b). The size of the vegetation clusters ranged from 0.12 to $250.0 \mathrm{~m}$, with a median cluster size of $20.0 \mathrm{~m}$ (Fig. 5c).

\subsubsection{Other features}

An additional 3 publications reported the detection of plastic litter using UAVs in the marine coastal and supratidal habitat. The size of plastic clusters surveyed was only provided in 2 publications, ranging from 0.02 to $0.60 \mathrm{~m}$, with a median size of $0.26 \mathrm{~m}$. Machine learning was also used in the 2 studies for which the size of plastic clusters was provided.

\subsubsection{Effect of region of interest size on use in machine learning}

The logistic regression models indicated no strong association between the size of a region of interest and the probability of machine learning being used to detect it. This pattern was consistent for each of 


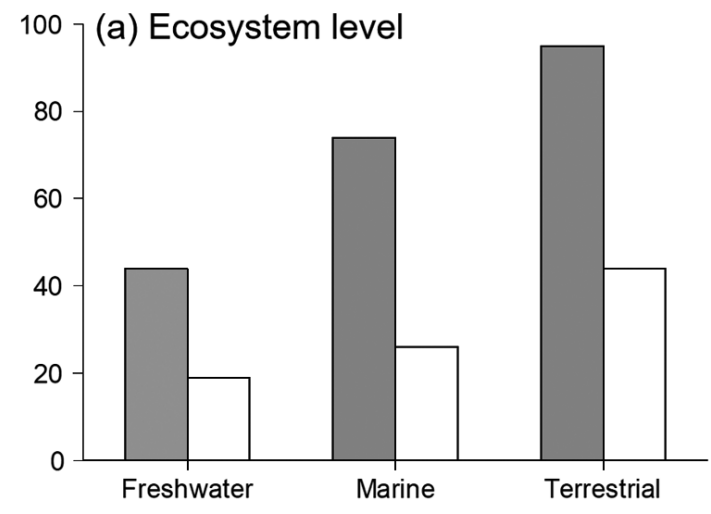

Fig. 3. Number of ecology publications in which UAVs have been used (grey bars) and the number of ecology publications in which machine learning has been used to process UAV imagery data (white bars) over the 2004-2018 period for (a) freshwater, marine and terrestrial ecosystems, (b) all animals and plants, (c) all animals only and (d) all vegetation only surveyed inthe 17 habitats as defined in the IUCN habitat classification scheme
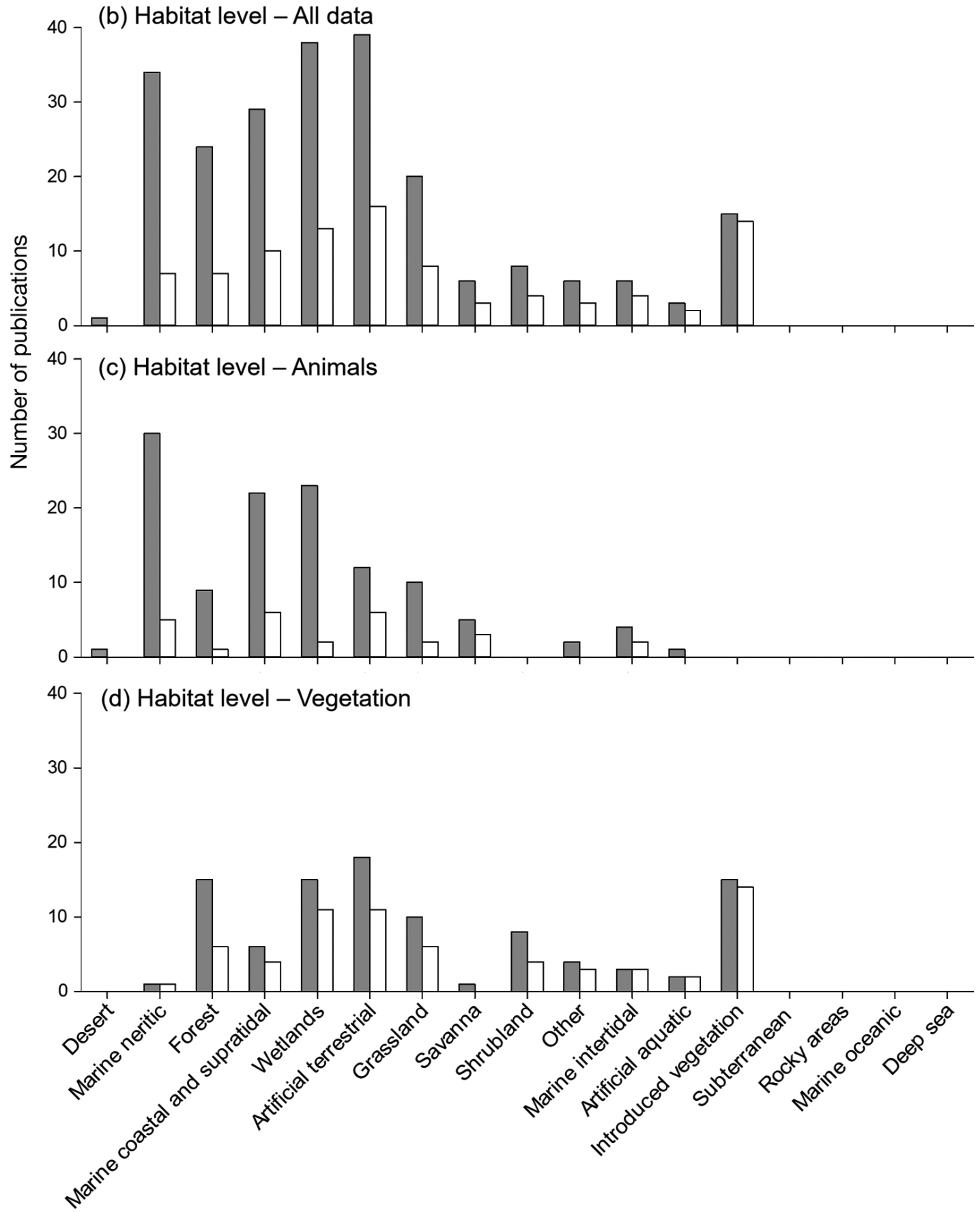

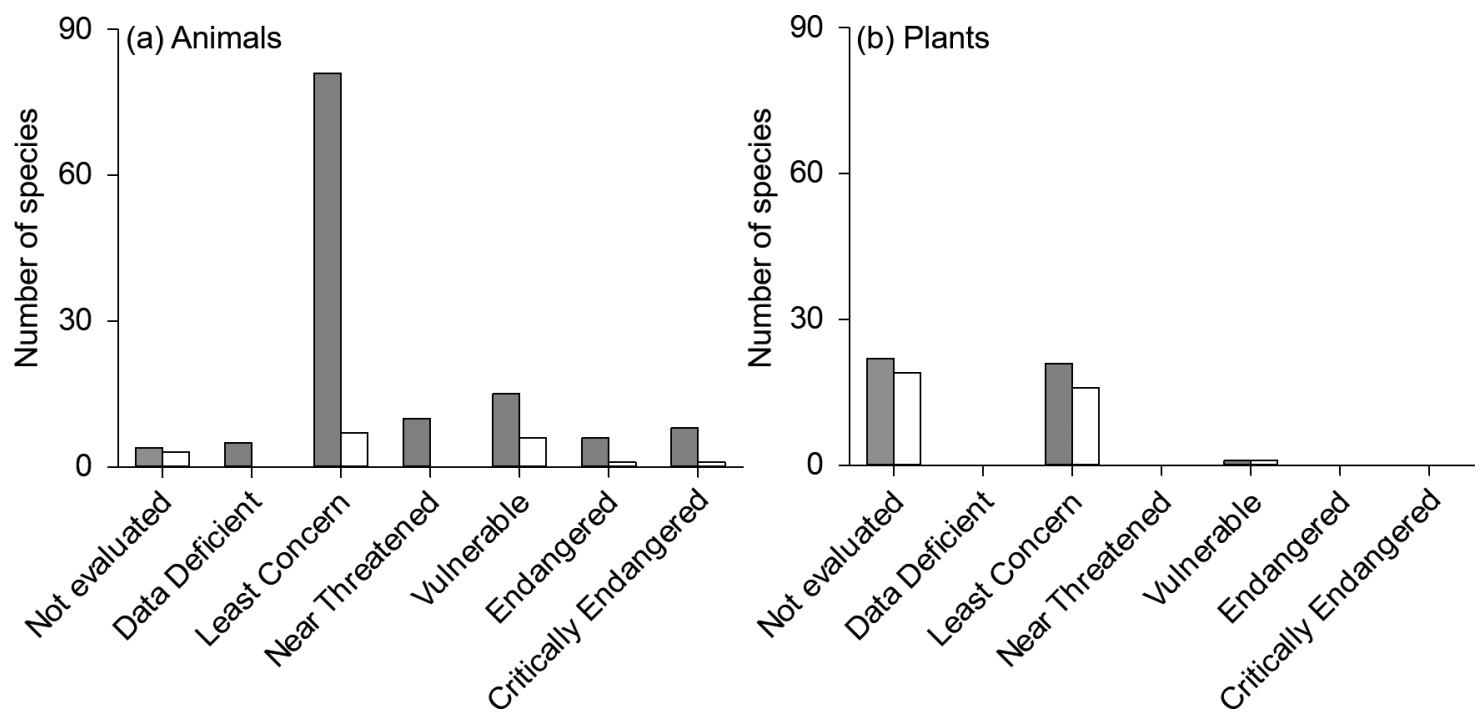

Fig. 4. Number of (a) animal and (b) plant species in each category of the IUCN Red Book detected using UAVs (grey bars) and the number of species detected using UAVs supported by machine learning (white bars)
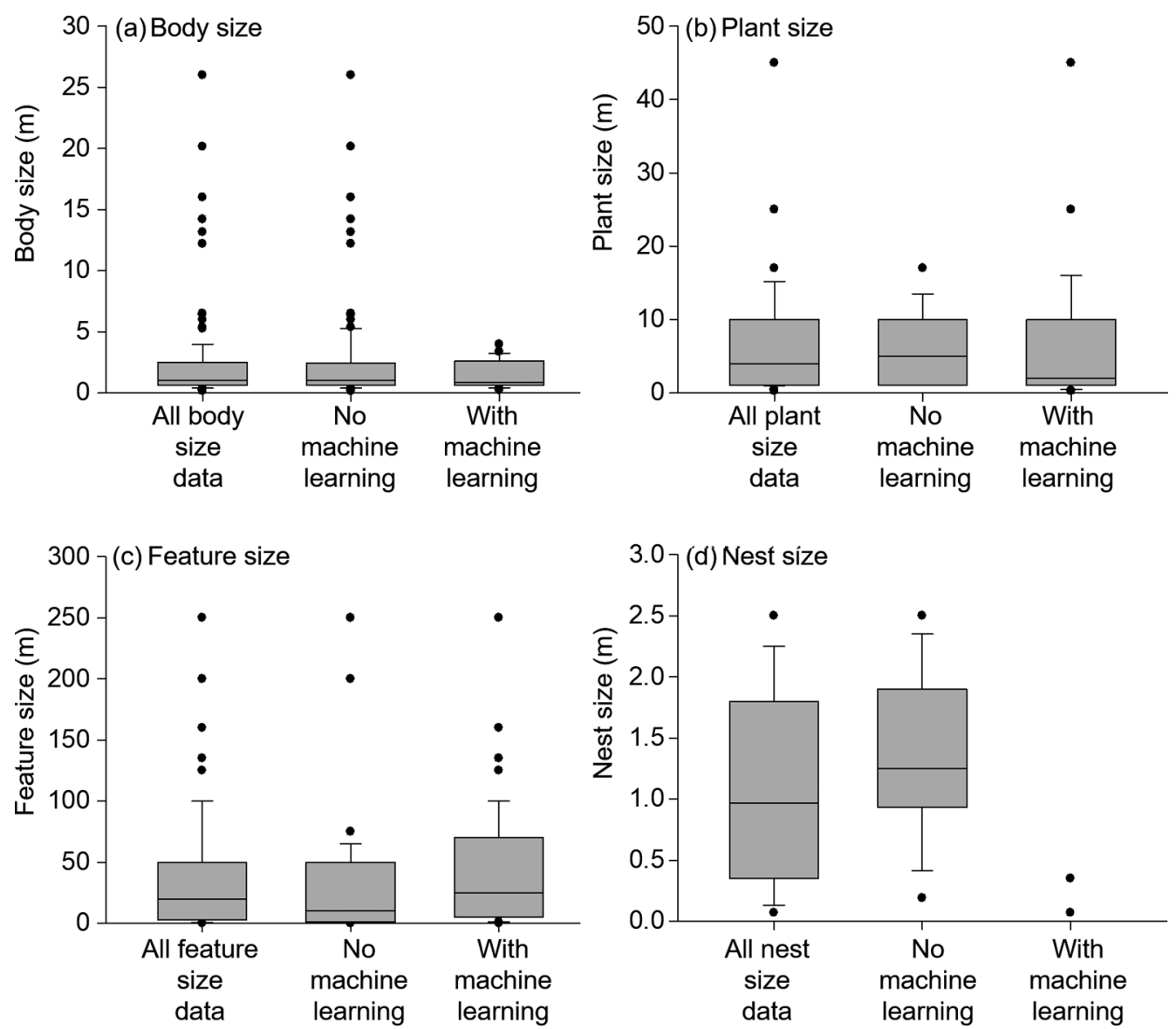

Fig. 5. Boxplots comparing the size of the regions of interest covered by UAVs in ecology publications in which machine learning was not used and in which machine learning was used for (a) body size of identified vertebrate and invertebrate individuals, (b) canopy diameter for individual plants, (c) feature size and (d) diameter of individual nests. Due to the small sample size, the nest size in publications with machine learning in (d) is represented as black dots. For all other boxplots, black dots: outliers; lower bound of the grey boxes: 1st quartile; upper bound of the grey boxes: 3rd quartile; middle horizontal black line: median; 
the 3 models separately fitted to animal body size, individual plant size and feature size. There was a significant relationship for nest size; however, the sample size was small with very large CI (Supplementary Results in Supplement 2). No relationship was observed when the 4 size categories were iteratively incorporated in the model or when the model was fitted using all 4 groupings (Supplementary Results in Supplement 2).

\subsection{Machine-learning methods used in ecology and across scientific fields}

Machine learning was used in nearly 90000 publications and in $>235$ scientific fields and subfields from 2004 to 2018 (Fig. 5). From the 213 publications using UAVs in ecology, 89 used machine learning, implementing 35 different machine-learning methods, of which 26 were used in a maximum 1 to 2 publications each (see Data in Supplement 1 for full list; Table 1, Fig. 6).

Of interest, the overall popularity of the different machine-learning methods compared to ecological studies using UAVs differed noticeably (Fig. 6). For instance, in ecology, object based image analysis (OBIA) was the most commonly encountered machine-learning method ( $\mathrm{n}=22$ publications), followed by random forests ( $\mathrm{n}=16$ publications), maximum likelihood classification ( $\mathrm{n}=13$ publications), support vector machine ( $\mathrm{n}=12$ publications), K-means clustering ( $\mathrm{n}=9$ publications), convolutional neural networks ( $\mathrm{n}=7$ publications) and finally thresholding ( $\mathrm{n}=5$ publications) (Table 1$)$. In comparison, for all scientific fields combined, support vector machine was the most commonly encountered machine-learning method ( 6000 publications in 2017) over the same period, followed by random forest and convolutional neural network ( 2500 publications in 2017). Thresholding ( 1700 publications in 2017) and K-means clustering had intermediate popularity $(\sim 1100$ publications in 2017). These differences might be associated with the specific application of these methods, rather than a more general purpose.

\section{DISCUSSION}

This study showed that, while the use of UAVs in ecological studies is expanding rapidly, the use of machine learning to survey the collected visual data is increasing at a much slower rate $(\sim 50 \%)$. This difference might be associated with the ability to detect features in habitats of varying complexity. For instance, UAVs are being used in almost every IUCN habitat category for both plant and animal surveys to distinguish regions of interest as small as $2 \mathrm{~cm}$. However, while machine-learning methods have been applied to distinguish similarly small features (e.g. small burrows), this tool has been used in fewer habitats and in ones that tend to exhibit greater uniformity, like artificial and aquatic habitats. For plants, machine learning is mostly used in heterogeneous habitats when assessing clusters of features, and a similar trend is observed when accessing clusters of invertebrates. Thus, machinelearning tools might not yet be accessible enough,
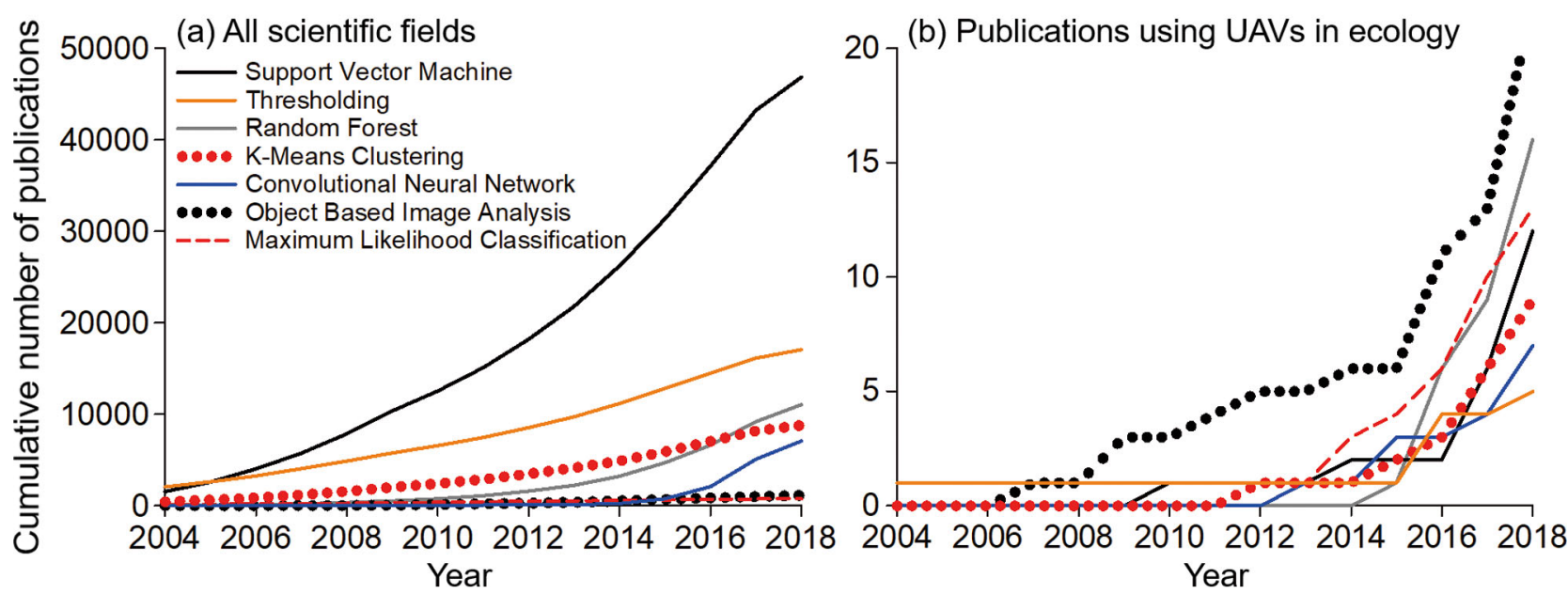

Fig. 6. Cumulative number of publications using 7 key machine-learning algorithms (see Table 1) across (a) all scientific fields and (b) for publications using UAVs in ecology for the 2004-2018 period 
and ecologists might not be familiar enough with them to efficiently replace the naked eye for assessing animals on heterogeneous backgrounds, which many natural habitats tend to be. Furthermore, at present, UAV and machine-learning studies are primarily led by institutions in countries with advanced economies, but this is likely to change as costs decline and algorithms are made freely available with publications (Watts et al. 2012, Christie et al. 2016). Six machine-learning methods are primarily used for processing UAV imagery in ecology at present, but these differ from the most popular methods used across the field of science in general; thus, potential methods that have not yet been fully trialled in ecology exist and have yet to be implemented to efficiently solve the issue of detecting animals in complex backgrounds.

\subsection{Geographical and temporal trends}

This study demonstrated that the rate of increase in the use of UAV technology in the field of ecology is $>2$ times greater than that for the adoption of machine learning to process the collected data. The ease of use of UAVs and their potential to complement a variety of existing field techniques means that the use of this technology will continue to spread and expand beyond economically advanced countries (Watts et al. 2012, Christie et al. 2016). The speed at which a new technology is adopted depends on a range of complex factors that are often difficult to quantify, such as the expectation for the technology to perform well and to be easy to use with low effort, user perception and the social environment (e.g. interactions within a research team) (Straub 2009, Wu et al. 2019). The adoption of machine learning is likely slower compared to the UAV technology because machine learning is more complex to implement and requires personnel with some degree of appropriate training, including computational and mathematical skills (see Hastie et al. 2009, Thessen 2016). As the number of available models and the autonomy of UAVs increases (i.e. batteries facilitate longer surveys), it is expected that routine flights will become common, covering larger spatial scales over longer time periods. This would solve issues of data availability by capturing a large quantity of imagery samples (Anderson \& Gaston 2013, Christie et al. 2016). Manual review of these datasets will become cost- and time-prohibitive and should prompt the adoption of machinelearning methods to improve efficiency. However, we also found that using machine learning alone does not increase the chances of an article being published in a higher impact journal; rather, insights on key ecological questions drive this phenomenon.

\subsection{Ecosystems, habitats, wildlife and plant species}

Our review showed that, while the institutions leading publications and the study sites are primarily based in economically advanced countries at present, UAV surveys have been conducted over most ecosystems and IUCN habitat categories, as well as on a range of species from different Red List categories. UAV surveys have even been conducted in habitats where other monitoring approaches are difficult to implement (such as using planes or helicopters in Antarctica; Nowacek et al. 2016, Johnston 2019), confirming the broad utility of integrating UAVs in monitoring and management (Anderson \& Gaston 2013, Christie et al. 2016). In particular, UAV surveys tended to be used differently between animal and vegetation. Specifically, UAV surveys tend be used differently for plants and animals. For instance, UAV surveys tend to be directed towards identifying individual animals rather than animal clusters but tend to be focused towards identifying vegetation clusters rather than individual plants. UAV surveys of individual animals, with and without machine learning, were primarily conducted in habitats with a background that was relatively uniform or homogeneous (e.g. freshwater or seawater areas, beaches or grasslands), with a limited amount of vegetation obstructing the field of view and potentially hiding animals. Plants were either detected in habitats with a high density of vegetation and a relatively complex background, with a focus on identifying clusters of vegetation with similar characteristics (e.g. using machine learning), or in habitats that were highly homogeneous with a background that was easy to process, with a focus on identifying individual plants. Therefore, we demonstrate that individual plants and individual animals were all generallydetected against a homogeneous background.

We found that the size of the regions of interest was not the main factor explaining why machine learning was used, with studies surveying regions of interest as small as $0.02 \mathrm{~m}$ and with $90 \%$ of regions of interest having a size ranging between 0.40 and $38.0 \mathrm{~m}$. Regardless of size, isolated individual animals or plants with a clearly defined shape and size against a homogeneous background tend to be relatively easy to 
detect by trained human observers. In such situations, the focus is often to obtain counts of individuals and determine the composition of species (e.g. Chabot et al. 2015, Hodgson \& Koh 2016, Rees et al. 2018). Such counts could be automated using machine learning, which would reduce the time-consuming process of manually reviewing the data. In comparison, it is more difficult to delineate clusters of dense vegetation or invertebrates, with $>1$ species often being present in a single cluster (e.g. Barrell \& Grant 2015, Michez et al. 2016). Because counts tend to be difficult to obtain within such clusters, scientists tend to focus on constructing distribution maps that are as precise as possible, rather than obtaining an exact count, which would require appropriate machine-learning methods to obtain high-quality results (Kaneko et al. 2014, Barrell \& Grant 2015, Michez et al. 2016).

We showed that both UAVs and machine learning are used to survey species classified as being Vulnerable, Endangered or Critically Endangered according to the IUCN Red List. However, machine learning was only used to process imagery data for a third of those same species. It might be easier to manually process imagery data over implementing a machinelearning model for rare and elusive species for which only a small amount of data are available (e.g. Schofield et al. 2017, Bonnin et al. 2018). However, as machine learning becomes more accessible and programs become available that can be adapted for different species, this tool will be invaluable for regular and repeated surveys of large geographical areas, or when the number of individuals is very large (Chabot et al. 2015, Bonnin et al. 2018, Hodgson et al. 2018). In addition, recent and successful attempts to detect endangered species using machine-learning algorithms (e.g. Gonzalez et al. 2016, Gray et al. 2018) suggest the use of these methods will increase with time.

\subsection{Machine-learning tools that are used to process UAV imagery data}

We observed that the popularity of certain machinelearning methods differs noticeably between ecology and other scientific fields. These differences probably reflect on the slow adoption of machine learning by ecologists (Thessen 2016) compared to other fields of biology such as medicine, for which the need to develop automatic and performant diagnostic tools has been a long-term goal (e.g. see Kononenko 2001) and for which large datasets are available for training algorithms. While machine learning is technical and time-consuming to learn, it needs to be incorporated in ecology studies to overcome the processing costs of increasing volumes of imagery data, including data from UAVs, BRUVs and camera traps. Once a machine learning model is successfully trained, processing newly collected data becomes relatively low-cost, easy to automate and suitable for long-term monitoring programs (Wagstaff 2012, Gonzalez et al. 2016, Tabak et al. 2018). In addition, machine-learning algorithms often outperform human observers, providing more accurate species count and distribution maps (e.g. Gray et al. 2018, Hodgson et al. 2018). The issue of background complexity potentially masking regions of interest could be resolved by using datasets of sufficient size containing samples of the region of interest over a range of different backgrounds, powerful computers and pre-trained deep convolutional neural networks. For a good example, see how Tabak et al. (2018) trained a convolutional neural network to identify species on camera trap images. However, such large datasets have often yet to be collected and shared with the community. This has probably contributed to the low use of machine learning with UAVs in ecology, to date, in addition to the fact that UAVs have only become commercially available (and hence accessible to many ecologists) over the last 5 yr (Anderson \& Gaston 2013, Christie et al. 2016).

While machine-learning methods can be complex to implement, effort is being made by machinelearning scientists to make these methods more accessible and facilitate transfer across scientific fields (Olden et al. 2008, Chicco 2017). Most of the methods summarized in Table 1 could be implemented in a range of statistical software on standard computers and laptops (except for complex deep convolutional neural networks, which require a dedicated and powerful computer). For example, the keras library may be used on both Python and R software and allows a convolutional neural network to be created and trained to classify imagery data in a few tenths of lines of code, which has greatly simplified the implementation of these models (Chollet 2015). In addition, increasing quantities of peerreviewed, grey literature and tutorials on how to implement machine-learning algorithms are now freely available online (Fawcett 2006, Olden et al. 2008, Domingos 2012, Chicco 2017).

In conclusion, for UAVs to be incorporated into cost-effective, regular and long-term monitoring protocols, parallel machine-learning methods are required to process the huge volumes of data generated, which are fast becoming too great for manual 
processing. At present, machine learning is being used to process certain types of features (such as small individuals or clusters of animals or plants) recorded by UAV surveys; however, issues with the complexity of the background must be overcome to generate efficient and indispensable machine-learning methods for use as part of the ecologist's tool box.

Author contributions. A.M.D. and G.S. designed the study. A.M.D. compiled and analysed the data. A.M.D. and G.S. wrote the manuscript.

\section{LITERATURE CITED}

Anderson K, Gaston KJ (2013) Lightweight unmanned aerial vehicles will revolutionize spatial ecology. Front Ecol Environ 11:138-146

Barasona JA, Mulero-Pázmány M, Acevedo P, Negro JJ, Torres MJ, Gortázar C, Vicente J (2014) Unmanned aircraft systems for studying spatial abundance of ungulates: relevance to spatial epidemiology. PLOS ONE 9: e115608

Barrell J, Grant J (2015) High-resolution, low-altitude aerial photography in physical geography: a case study characterizing eelgrass (Zostera marina L.) and blue mussel (Mytilus edulis L.) landscape mosaic structure. Prog Phys Geogr 39:440-459

Blaschke T (2010) Object based image analysis for remote sensing. ISPRS J Photogramm Remote Sens 65:2-16

* Bonnin N, Van Andel A, Kerby J, Piel A, Pintea L, Wich S (2018) Assessment of chimpanzee nest detectability in drone-acquired images. Drones 2:17

* Chabot D, Craik SR, Bird DM (2015) Population census of a large common tern colony with a small unmanned aircraft. PLOS ONE 10:e0122588

Chicco D (2017) Ten quick tips for machine learning in computational biology. BioData Min 10:1-17

* Chollet F (2015) Keras. Github. https://github.com/fchollet/ keras

Christiansen F, Dujon AM, Sprogis KR, Arnould JPY, Bejder L (2016) Noninvasive unmanned aerial vehicle provides estimates of the energetic cost of reproduction in humpback whales. Ecosphere 7:e01468

Christie KS, Gilbert SL, Brown CL, Hatfield M, Hanson L (2016) Unmanned aircraft systems in wildlife research: current and future applications of a transformative technology. Front Ecol Environ 14:241-251

* Cutler DR, Edwards TC, Beard KH, Cutler A, Hess KT, Gibson J, Lawler JJ (2007) Random forests for classification in ecology. Ecology 88:2783-2792

Dell AI, Bender JA, Branson K, Couzin ID and others (2014) Automated image-based tracking and its application in ecology. Trends Ecol Evol 29:417-428

Domingos P (2012) A few useful things to know about machine learning. Commun ACM 55:78-87

Duffy MA (2017) Last and corresponding authorship practices in ecology. Ecol Evol 7:8876-8887

Egnor SER, Branson K (2016) Computational analysis of behavior. Annu Rev Neurosci 39:217-236

Fawcett T (2006) An introduction to ROC analysis. Pattern Recognit Lett 27:861-874

Foody GM, Campbell NA, Trodd NM, Wood TF (1992) Deri- vation and applications of probabilistic measures of class membership from the maximum-likelihood classification. Photogramm Eng Remote Sensing 58:1335-1341

* Gonzalez LF, Montes GA, Puig E, Johnson S, Mengersen K, Gaston KJ (2016) Unmanned aerial vehicles (UAVs) and artificial intelligence revolutionizing wildlife monitoring and conservation. Sensors (Basel) 16:97

* Gray PC, Fleishman AB, Klein DJ, McKown MW, Bézy VS, Lohmann KJ, Johnston DW (2018) A convolutional neural network for detecting sea turtles in drone imagery. Methods Ecol Evol 10:345-355

* Guilford T, Meade J, Willis J, Phillips RA and others (2009) Migration and stopover in a small pelagic seabird, the Manx shearwater Puffinus puffinus: insights from machine learning. Proc Biol Sci 276:1215-1223

Hastie T, Tibshirani R, Friedman J (2009) The elements of statistical learning, 2nd edn. Springer-Verlag, New York, NY

*Hodgson JC, Koh LP (2016) Best practice for minimising unmanned aerial vehicle disturbance to wildlife in biological field research. Curr Biol 26:R404-R405

*Hodgson JC, Mott R, Baylis SM, Pham TT and others (2018) Drones count wildlife more accurately and precisely than humans. Methods Ecol Evol 9:1160-1167

Hsu CW, Chang CC, Chih-Jen L (2008) A practical guide to support vector classification. Technical report, Department of Computer Science, National Taiwan University

International Monetary Fund (2016) World Economic Outlook: too slow for too long. IMF, Washington, DC. www.imf.org/external/pubs/ft/weo/2016/01/

International Union for Conservation of Nature (2012) Habitats classification scheme (Version 3.1). IUCN, Gland. https://www.iucnredlist.org/resources/habitat-classifi cation-scheme

* Johnston DW (2019) Unoccupied aircraft systems in marine science and conservation. Annu Rev Mar Sci 11:439-463

*Jordan MI, Mitchell TM (2015) Machine learning: trends, perspectives, and prospects. Science 349:255-260

*Kampichler C, Wieland $\mathrm{R}$, Calmé S, Weissenberger $H_{\text {, }}$ Arriaga-Weiss S (2010) Classification in conservation biology: a comparison of five machine-learning methods. Ecol Inform 5:441-450

Kaneko K, Nohara S, Kaneko K, Nohara S (2014) Review of effective vegetation mapping using the UAV (Unmanned Aerial Vehicle) method. J Geogr Inf Syst 6:733-742

Koh LP, Wich SA (2012) Dawn of drone ecology: low-cost autonomous aerial vehicles for conservation. Trop Conserv Sci 5:121-132

Kononenko I (2001) Machine learning for medical diagnosis: history, state of the art and perspective historical overview. Artif Intell Med 23:89-109

*L'Heureux A, Grolinger K, Elyamany HF, Capretz MAM (2017) Machine learning with big data: challenges and approaches. IEEE Access 5:7776-7797

*Mallet D, Pelletier D (2014) Underwater video techniques for observing coastal marine biodiversity: a review of sixty years of publications (1952-2012). Fish Res 154: 44-62

* Mazloumian A, Helbing D, Lozano S, Light RP, Börner K (2013) Global multi-level analysis of the 'scientific food web.'. Sci Rep 3:1-5

'Michez A, Piégay H, Jonathan L, Claessens H, Lejeune P (2016) Mapping of riparian invasive species with supervised classification of unmanned aerial system (UAS) imagery. Int J Appl Earth Obs Geoinf 44:88-94 
Mountrakis G, Im J, Ogole C (2011) Support vector machines in remote sensing: a review. ISPRS J Photogramm Remote Sens 66:247-259

Mulero-Pázmány M, Stolper R, Van Essen LD, Negro JJ, Sassen T (2014) Remotely piloted aircraft systems as a rhinoceros anti-poaching tool in Africa. PLOS ONE 9: e83873

Nowacek DP, Christiansen F, Bejder L, Goldbogen JA, Friedlaender AS (2016) Studying cetacean behaviour: new technological approaches and conservation applications. Anim Behav 120:235-244

Olden JD, Lawler JJ, Poff NL (2008) Machine learning methods without tears: a primer for ecologists. Q Rev Biol 83: 171-193

Olivares-Mendez MA, Fu C, Ludivig P, Bissyandé TF and others (2015) Towards an autonomous vision-based unmanned aerial system against wildlife poachers. Sensors (Basel) 15:31362-31391

Parry DM, Kendall MA, Pilgrim DA, Jones MB (2003) Identification of patch structure within marine benthic landscapes using a remotely operated vehicle. J Exp Mar Bio Ecol 286:497-511

QGIS Development Team (2015) QGIS Geographic Information System. Open Source Geospatial Foundation Project. http://qgis.osgeo.org.

R Development Core Team (2013) R: a language and environment for statistical computing. R Foundation for Statistical Computing, Vienna

Rees AF, Avens L, Ballorain K, Bevan E and others (2018) The potential of unmanned aerial systems for sea turtle research and conservation: a review and future directions. Endang Species Res 35:81-100

Schofield G, Katselidis KA, Lilley MKS, Reina RD, Hays GC (2017) Detecting elusive aspects of wildlife ecology using drones: new insights on the mating dynamics and operational sex ratios of sea turtles. Funct Ecol 31:2310-2319

Stan Development Team (2018) RStan: the R interface to Stan. http://mc-stan.org/

Straub ET (2009) Understanding technology adoption: theory and future directions for informal learning. Rev Educ Res 79:625-649

Editorial responsibility: Alan F. Rees (Guest Editor), Exeter, UK
Strobl C, Malley J, Gerhard T (2009) Characteristics of classification and regression trees, bagging and random forests. Psychol Methods 14:323-348

Szantoi Z, Smith SE, Strona G, Koh LP, Wich SA (2017) Mapping orangutan habitat and agricultural areas using Landsat OLI imagery augmented with unmanned aircraft system aerial photography. Int J Remote Sens 38: $2231-2245$

Tabak MA, Norouzzadeh MS, Wolfson DW, Sweeney SJ and others (2018) Machine learning to classify animal species in camera trap images: applications in ecology. Methods Ecol Evol 10:585-590

* Tarca AL, Carey VJ, Chen X, Romero R, Dr ghici S (2007) Machine learning and its applications to biology. PLOS Comput Biol 3:e116

Thessen A (2016) Adoption of machine learning techniques in ecology and earth science. One Ecosystem 1:e8621

* Trathan PN (2004) Image analysis of color aerial photography to estimate penguin population size. Wildl Soc Bull 32:332-343

United Nations Educational Scientific and Cultural Organization (2016) UNESCO science report: toward 2030. UNESCO SC.2015/WS/24

Wagstaff K (2012) Machine learning that matters. In: Langford J, Pineau L (eds) Proc 29th International Conference on Machine Learning, Edinburgh, Scotland, June 26July 1, 2012, p 1-6

Watts AC, Ambrosia VG, Hinkley EA (2012) Unmanned aircraft systems in remote sensing and scientific research: classification and considerations of use. Remote Sens 4: 1671-1692

Weinstein BG (2018) A computer vision for animal ecology. J Anim Ecol 87:533-545

$\mathrm{Wu} \mathrm{J}$ (2017) Introduction to convolutional neural networks. Nanjing University

W Wu L, Wang D, Evans JA (2019) Large teams develop and small teams disrupt science and technology. Nature 566: 378-382

Zou Q, Xie S, Lin Z, Wu M, Ju Y (2016) Finding the best classification threshold in imbalanced classification. Big Data Res 5:2-8

Submitted: December 17, 2018; Accepted: March 28, 2019 Proofs received from author(s): June 5, 2019 